\title{
SARS-CoV 蛋白质组的生物信息学及其进化关系
}

\author{
柳树群 ${ }^{*}$ 过 涛” 季星来 孙之荣
}

(清华大学生物科学与技术系, 生物信息学研究所, 北京 100084. * 同等贡献.†联系人, E-mail: sunzhr@mail.tsinghua.edu.cn)

\begin{abstract}
摘要 一种新的冠状病毒 SARS-CoV 是引起严重急性呼吸综合征(severe acute respiratory syndrome, SARS)的病原体。对由 SARS 病毒全基因组序列推导出的所有蛋白质逐一进行分子量、等电点、分子消 光系数等物理化学性质计算, 以及跨膜区和亚细胞定位预测, 辅以保守序列家族数据库搜索, 预测 SARS-CoV 功能未知蛋白质的功能. 同时, 通过 SARS-CoV 与其他冠状病毒蛋白质同源序列比较和进化 距离计算, 分析 SARS 病毒的分类地位以及与其他冠状病毒的进化关系. 结果表明, 尽管 SARS 病毒是 不同于其他 3 组冠状病毒的一种全新冠状病毒, 但在进化关系上更靠近牛冠状病毒 BoCoV 和鼠肝炎病 毒 MHV. 为实验测定 SARS 病毒蛋白质组以及抗 SARS 疫苗研制提供了参考和帮助.
\end{abstract}

\section{关键词 SARS SARS-CoV 保守序列家族 序列比较 进化}

2002 年 11 月, 第 1 例严重急性呼吸综合征(severe acute respiratory syndrome, SARS)病例在中国广东省 发现. 之后, SARS 传播至香港、越南、新加坡、加拿 大等 31 个国家和地区. 截止 2003 年 5 月 28 日, 全球 累计 8221 人被感染, 死亡 735 人, 其中中国内地累计 5332 人被感染, 死亡 321 人, 死亡率由世界卫生组织 (World Health Organization, WHO)最初公布的约 4\% 上升到约 15\%(http://www.who.int/csr/sars/).

2003 年 4 月, 世界卫生组织宣布, 在中国、德国、 加拿大等 10 个国家和地区的 13 个实验室的共同努力 下, 正式确认一种新的冠状病毒 (Coronavirus) 为引起 SARS 的病原体, 并将其命名为 SARS 冠状病毒 $(\mathrm{SARS}-\mathrm{CoV})^{[1 \sim 3]}$. 加拿大、中国内地及香港、美国和 新加坡的科学家相继公布了他们测定的 SARS 病毒 基因组 ${ }^{[3 \sim 7]}$. 截止 2003 年 5 月 30 日, NCBI 的 GenBank 数据库 (http://www.ncbi.nlm.nih.gov/) 已经收录了 SARS 病毒相关核酸序列 27 条, 其中完整的 SARS 病 毒基因组 14 个.

冠状病毒属于 Nidovirales 目 Coronaviridae 科 Coronavirus 属, 它寄生于人类和其他动物的细胞质 内, 能够引起宿主呼吸系统和消化系统疾病. 这种病 毒含有一条约 30000 个核苷酸长的正链 RNA, 是已 知的拥有最大基因组的 RNA 病毒. 目前发现的冠状 病毒按血清型可分为 3 个组, 第 1 组和第 2 组包含哺 乳动物冠状病毒, 而第 3 组只包含鸟类冠状病毒. 在 每一组中, 依据病毒侵染的宿主范围、抗原关联程度 以及基因组的组织形式, 冠状病毒被分为不同的种. 每一种冠状病毒都只能侵染特定的宿主, i同时病毒
对体外培养的条件要求相当苛刻. 冠状病毒能够引 起很多动物的严重传染性疾病, 如禽支气管炎、猫腹 膜炎、猪肠胃炎等，常常造成畜牧业的巨大损失 ${ }^{[8]}$. 以前发现的两种人类冠状病毒 HCoV-229E 和 HCoVOC43 分别属于第 1 类和第 2 类冠状病毒, 人类约 $30 \%$ 的流行性感冒和上呼吸道感染是由这两种病毒 引起的 ${ }^{[9]}$. 冠状病毒一旦进人细胞, 其 RNA 的 $5^{\prime}$ 端可 读框 ORF $1 \mathrm{ab}$ 即被翻译成一条很长的多蛋白体链 (polyprotein), 该多蛋白体被病毒自身编码的蛋白酶 水解为多个蛋白质产物 ${ }^{[5]}$, 其中包括 3C-like 蛋白酶 $\left(3 \mathrm{CL}^{\text {pro }}\right.$, chymotrypsin-like proteinase)、RNA 依赖的 RNA 聚合酶(Rep, RNA-dependent RNA polymerase) 以及 ATP 螺旋酶(Hel, ATPase-Helicase)等. Rep 和 Hel 负责病毒基因组的复制和转录, 病毒 mRNA 被宿主 细胞的核糖体翻译成各种病毒蛋白质. 其中包括 4 种 主要结构蛋白质(structural protein): 核壳体蛋白 $\mathrm{N}$ (nucleocapsid)、刺突蛋白 S (spike)、膜蛋白 M (membrane) 和小包被蛋白 $\mathrm{E}$ (small envelope), 它们全部为糖蛋白 (glycoprotein). 新复制出的全长正链 RNA 首先与核 壳体蛋白 $\mathrm{N}$ 结合形成 RNA-蛋白质复合物, 然后该复 合物与位于内质网膜上的 $\mathrm{M}$ 蛋白和 $\mathrm{E}$ 蛋白装配形成 新的病毒颗粒, 病毒颗粒被内质网膜出芽的小囊泡 运送到高尔基体, 最终以类似于胞分泌的形式释放 出细胞 ${ }^{[10]}$. 需要指出的是, 位于病毒膜上的 $\mathrm{S}$ 蛋白是 决定病毒宿主和组织特异性的关键蛋白质, 因为病 毒是通过 $\mathrm{S}$ 蛋白与细胞表面的特殊受体相互作用而 侵人细胞的 ${ }^{[8]}$.

10.136 目前 NCBI GenBank 数据库收录的 14 条完整 
SARS-CoV 基因组序列, 长度变化范围为 29712 29751 个核苷酸, 序列差异约为 $0.1 \%$, 整个基因组的 G-C 含量约为 $41 \%$, SARS-CoV 基因组的组织形式与 其他冠状病毒相同, 从 $5^{\prime}$ 端开始依次为复制酶基因 rep, 刺突蛋白基因 $S$, 小包被蛋白基因 $E$, 膜蛋白基 因 $M$, 核壳体蛋白基因 $N^{[5,6]}$. 其中 rep 基因约占整个 基因组的 2/3, 它包含可读框 ORF1a 和 ORF1b, 这两 个 ORF 经过共翻译水解而产生大量蛋白质产物. 同 时, $S$ 基因与 $E$ 基因之间, $M$ 与 $N$ 之间以及 $N$ 基因下 游的区域编码一些非结构蛋白质(non-structural protein), 这些非结构蛋白在不同的冠状病毒之间差别很 大, 功能还不清楚 ${ }^{[4 \sim 6]}$.

本研究利用生物信息学手段, 对所有 SARS-CoV 相关蛋白质进行分子量、等电点、分子消光系数等物 理化学性质计算以及跨膜螺旋区和亚细胞定位预测, 同时对每个 SARS-CoV 蛋白质进行保守序列家族和 同源蛋白质序列搜索, 进一步对 SARS-CoV 的蛋白 质与其他冠状病毒的对应蛋白质进行了进化距离计 算和分析.

\section{1 材料与方法}

在蛋白质序列数据库 SWISS-PROT(http://www. ebi.ac.uk/swissprot/) 中以 “SARS”为关键词搜索 SARS-CoV 相关的蛋白质序列. 由于 SWISS-PROT数 据库中收录的 SARS-CoV 相关蛋白质序列参考了 GenBank 数据库中对应的所有核酸序列, 因此, 来自 SWISS-PROT 数据库的蛋白质序列比 GenBank 的蛋 白质序列更具有代表性 ${ }^{[11]}$. SWISS-PROT 数据库收录 了 10 个 SARS-CoV 相关蛋白质, 包括可读框 ORF1ab 对应的蛋白产物, 结构蛋白 $\mathrm{S}, \mathrm{E}, \mathrm{M}, \mathrm{N}$ 以及功能未知 的蛋白 X1 X4. 将这些蛋白质与 GenBank 中编号为 AY274119 的 SARS-CoV 全基因组的可读框一一进行 比较, 发现可读框 ORF9 11 和 ORF 14 编码的蛋白质 没有被 SWISS-PROT 数据库收录. 所有 SARS-CoV 相关蛋白质(包括 SWISS-PROT 收录的蛋白质以及由 SARS-CoV 基因组 ORF 推测出的蛋白质)列于表 1 . 对其他冠状病毒的研究表明 ${ }^{[12]}$, 可读框 ORF1ab 占据 了约 $2 / 3$ 的基因组长度, 具体分为两个阅读框 ORF1a 和 ORF1b, 当核糖体翻译到 ORF1a 和 ORF1b 交界处 13392 13398(5' -UUUAAAC-3') 位置时, 核糖体对
ORF1b 进行了 -1 位的移框阅读, 同时在 ORF1a 和 ORF1b 翻译过程中, 翻译出来的多蛋白质体经历了 一系列水解过程, 首先得到 PLP ${ }^{\text {pro }}$ (papain-like proteinase)和 $3 \mathrm{CL}^{\mathrm{pro}}$, 接着生成了包括 Rep 和 $\mathrm{Hel}$ 在内的 一系列蛋白质产物. 通过对 SARS-CoV 可读框 ORF1ab 的切割位点分析, 共得到 14 个蛋白产物(表 2), 由于 这些蛋白产物真正存在于病毒的侵染周期中, 我们 对这 14 个蛋白质而不是对 ORF1ab 对应的整条蛋白 序列进行了生物信息学计算和分析.

首先, 用 SAPS(statistical analysis of protein sequence)程序 ${ }^{[13]}$ 统计每个 SARS-CoV 蛋白质的氨基酸 组成、可能出现的重复序列模式以及电荷分布, 用 PI 程序 ${ }^{1}{ }^{1}$ 和 EXTCOEF(extinction coefficient calculation) 程序 ${ }^{[14]}$ 分别计算每个蛋白质的等电点和消光系数, 用 TMAP ${ }^{[15]}$ 和 TMHMM $2.0^{[16]}$ 程序预测蛋白质的跨膜 片段和跨膜螺旋区，以确定蛋白质可能的亚细胞定 位. 用 HMMPFAM ${ }^{[17]}$ 和 BLIMPS ${ }^{[18]}$ 方法分别在 HMM 数据库和 BLOCKS 数据库中搜索可能匹配的蛋白质 保守序列家族. 最后, 利用 Workbench 生物信息学平 台 ${ }^{[14]}$ 提供的 PSIBLAST 程序 ${ }^{[19]}$ 在非冗长蛋白质序列 数据库(non-redundant protein database(SDSC))中搜索每 个 SARS-CoV 相关蛋白的同源序列(选择 BLOSUM80 矩阵, 最大迭代次数 6), 并用 CLUSTALW 程序 ${ }^{[20]}$ 对 搜索到的同源序列进行多序列比对, 根据比对结果, 用 DRAWTREE 和 DRAWGRAM ${ }^{[21]}$ 绘制进化树, 并使 用 PROTDIST 程序 ${ }^{2}$ 计算蛋白质序列之间的进化距 离矩阵, 以期阐明 SARS-CoV 与其他冠状病毒之间 的进化关系.

\section{2 结果和讨论}

与其他冠状病毒一样, SARS-CoV 的蛋白质分为 结构蛋白和非结构蛋白, 前者如 S, E, M, N 等, 它们 是病毒蛋白质外壳的组成成分, 后者如 $3 \mathrm{CL}^{\mathrm{pro}}$, Rep 和 Hel 等, 分别负责病毒蛋白质的水解和病毒 RNA 的复制和转录. 此外, SARS-CoV 还包含大量的功能 未知蛋白(function unknown protein), 这些蛋白是由 病毒基因组序列的可读框 $\mathrm{ORF}$ 推测出来的, 如 $\mathrm{X} 1$, $\mathrm{X} 2, \mathrm{X} 3, \mathrm{X} 4, \mathrm{X} 5$ 等. 本文对所有 SARS-CoV 的相关蛋 白质进行了生物信息学计算和分析, 每个蛋白质的 序列长度、分子量、消光系数以及等电点等物理化学

1) Program by Dr Luca Toldo, developed at http://www.embl-heidelberg.de

2) Felsenstein J. PHYLIP (Phylogeny Inference Package). Version 3.5c. 199360/csb2003-48-13-1359 
性质列于表 1 和表 2.

\subsection{ORF1ab 编码的蛋白质产物分析}

可读框 ORF1ab 编码的多蛋白体经过共翻译水 解过程 (con-translational proteolytic processing), 产生 约 14 个蛋白产物(表 2). 其中前导蛋白(LP, leader protein) 是一个分子量为 $19.6 \mathrm{kD}$ 的酸性蛋白质, 正电 氨基酸(KR)占 $10.1 \%$, 负电氨基酸(ED)占 $14.5 \%$, TMAP 和 TMHMM 预测没有发现该蛋白质中存在任何跨膜 片段. BLIMPS 搜索发现, 前导蛋白中 25 49 肽段与 BLOCKS 数据库中 IPB001407D 保守序列模式相匹 配, IPB001407D 来自流感病毒的 RNA 依赖的 RNA 聚合酶. PSIBLAST 搜索发现, 前导蛋白中部分序列 片段分别与内因子-维生素 $\mathrm{B}_{12}$ 受体(intrinsic factor- vitamin $\mathrm{B}_{12}$ receptor)、维生素 $\mathrm{B}_{12}$ 的受体蛋白质 cubilin、阳离子转运蛋白/ATP 酶(cation transporter/ ATPase)、氨肽酶(amino- peptidase N)等蛋白质的部分 序列片段有 $30 \%$ 左右的同源性.

P65 是分子量为 $70.7 \mathrm{kD}$ 的弱酸性蛋白, TMAP 预 测 400 424, 467 486 位置存在两个潜在的跨膜片段, 而 TMHMM 没有预测出任何跨膜螺旋片段. BLIMPS 搜索发现 508 545 肽段与 IPB001156 (transferring, 转 铁蛋白)保守序列家族相匹配, HMMPFAM 搜索发现 142 185 和 291 351 片段分别与 HMM 数据库中的 PF05256(uncharacterised protein family, 无典型特征 的蛋白质家族)和 PF00087 (snake toxin, 蛇毒蛋白)匹 配. PSIBLAST 搜索发现, P65 的部分序列分别与黄果

表 1 SARS-CoV 相关蛋白质的物理化学性质

\begin{tabular}{|c|c|c|c|c|c|c|}
\hline 蛋白质 & 数据库登录号 ${ }^{\mathrm{b})}$ & 长度 $(\mathrm{aa})$ & 分子量/kD & Extcoef $^{\mathrm{c})} /(\mathrm{mol} / \mathrm{L})^{-1} \cdot \mathrm{cm}^{-1}$ & $\mathrm{pI}^{\mathrm{d})}$ & 功能分类 \\
\hline ORF1ab & P59641 & 7073 & 790.3 & 866830 & 6.2 & 多蛋白质体 \\
\hline S & P59594 & 1255 & 139.1 & 134050 & 5.4 & 结构蛋白 \\
\hline $\mathrm{X} 1$ & P59632 & 274 & 30.9 & 50690 & 5.7 & 结构蛋白 ${ }^{\mathrm{e})}$ \\
\hline $\mathrm{X} 2$ & P59633 & 154 & 17.8 & 9770 & 11.2 & 非结构蛋白 ${ }^{\mathrm{e})}$ \\
\hline $\mathrm{E}$ & P59637 & 76 & 8.3 & 5300 & 6.3 & 结构蛋白 \\
\hline M & P59596 & 221 & 25.1 & 51530 & 9.8 & 结构蛋白 \\
\hline ORF9 & AY274119 & 44 & 5.3 & 7079 & 3.4 & 结构蛋白 ${ }^{\text {) }}$ \\
\hline ORF10 & AY274119 & 39 & 4.3 & 360 & 8.0 & N/A ${ }^{f)}$ \\
\hline $\mathrm{X} 5$ & AY274119 & 84 & 9.6 & 21210 & 9.5 & 非结构蛋白 ${ }^{\mathrm{e}}$ \\
\hline $\mathrm{N}$ & P59595 & 422 & 46.0 & 42530 & 10.5 & 结构蛋白 \\
\hline HP5 ${ }^{\text {a) }}$ & P59636 & 98 & 10.8 & 1280 & 4.7 & $\left.N / A^{f}\right)$ \\
\hline
\end{tabular}

a) 假设的蛋白质 5 ( hypothetical protein 5);b) P596xx 为 Swiss-Prot 数据库编号, AY274119 为 GenBank 数据库编号; c) Extcoef 为 $280 \mathrm{~nm}$ 波长下的分子消光系数; d) pI 为等电点; e) 本文推测的蛋白质可能的功能(详见正文); f) 蛋白质的功能未知 (function not available)

表 2 可读框 ORF1ab 推测出来的蛋白质产物的物理化学性质

\begin{tabular}{|c|c|c|c|c|c|c|}
\hline ORF 产物 & ORF & 起始位置 & 长度(aa) & 分子量/kD & Extcoef $/(\mathrm{mol} / \mathrm{L})^{-1} \cdot \mathrm{cm}^{-1}$ & $\mathrm{pI}$ \\
\hline LP & ORF1a & $1 \sim 179$ & 179 & 19.6 & 13370 & 5.2 \\
\hline $\mathrm{P} 65^{\text {a) }}$ & ORF1a & $180 \sim 818$ & 639 & 70.7 & 63150 & 6.0 \\
\hline PLP & ORF1a & $819 \sim 3239$ & 2421 & 269.7 & 28110 & 5.4 \\
\hline HD2/NSP3 & ORF1a & $3548 \sim 3836$ & 289 & 33.0 & 59180 & 8.9 \\
\hline NSP4 ${ }^{b)}$ & ORF1a & $3837 \sim 3919$ & 83 & 9.3 & 5870 & 5.0 \\
\hline GFLP & ORF1a & $4230 \sim 4369$ & 140 & 14.8 & 12870 & 6.3 \\
\hline Rep & ORF1a, ORF1b & $4370 \sim 5301$ & 932 & 106.5 & 127310 & 6.0 \\
\hline Hel & ORF1b & $5302 \sim 5902$ & 601 & 66.9 & 62150 & 8.2 \\
\hline NSP11 & ORF1b & $5903 \sim 6429$ & 527 & 59.9 & 89000 & 7.3 \\
\hline NSP12 & ORF1b & $6430 \sim 6775$ & 346 & 38.5 & 31450 & 4.9 \\
\hline
\end{tabular}

a) 该蛋白对应于鼠肝炎病毒 MHV 的 P65 蛋白, b) NSP 表示功能未知蛋白质 
蝇(Drosophila melanogaster), 细胞膜外蛋白质 B (extracellular matrix protein B), 线粒体内的核糖体蛋白 (mitochondrial ribosomal protein), 假设的 $12.7 \mathrm{kD}$ 的 蛋白质(其编码区位于 $16 \mathrm{~S}$ 和 $23 \mathrm{~S}$ RNA 编码 DNA 之 间, Hypothetical $12.7 \mathrm{kD}$ protein in 16S-23S DNA spacer)以及无害利斯特菌(Listeria innocua)等蛋白的 部分序列片段有 $30 \%$ 左右的同源性.

PLP ${ }^{\text {pro }}$ 蛋白质是分子量为 $269.7 \mathrm{kD}$ 的强酸性蛋 白质, 含有丰富的半胱氨酸(3\%), 其中 111 183 序列 DEEEEDDAECEEEEIDETCEHEYGTEDDYQGLPLEFGASAETVRVEEEEEEDWLDDTTEQSEIEPEPEPTPEE 为强负电区域. TMAP 和 TMHMM 预测出 PLP $\mathrm{P}^{\text {pro }}$ 含有 7 个跨膜螺旋片段, 而 $\mathrm{N}$ 末端相当长的一段区域 1 1390 位于病毒粒子外部或内质网/高尔基体(ER/ Golgi)内部, $\mathrm{C}$ 末端 112 个氨基酸残基位于病毒粒子 内部或细胞质内. HMMPFAM 分析发现 PLP ${ }^{\text {pro }} 44 \sim 182$, 218 326, 790 1065, 1216 1416, 2152 2312 区域分别 与保守序列家族 PF05066(RNA_pol_delta)、PF01661 (A1pp)、PF01831 (peptidase_C16)、PF03649(UPF0014)、 PF01891 (CbiM)相匹配. BLIMPS 搜索发现 PLP ${ }^{\text {pro }}$ 的 部分序列分别与 BLOCKS 数据库中的保守蛋白序列 IPB002589 (domain of unknown function DUF27, 功 能未知结构域 DUF27)、IPB001509 (NAD dependent epimerase/dehydratase family, NAD 依赖的差向异构 酶/脱水酶)、IPB001394 (ubiquitin carboxyl-terminal hydrolase family 2, 泛素羧端水解酶家族)、IPB000135 (high mobility group proteins HMG1 and HMG2, 高迁 移率组分蛋白质 HMG1 与 HMG2)、PR00375 (Huntingtin signature, Huntingtin 舞蹈症蛋白质信号)相匹 配. PSIBLAST 搜索表明, SARS-CoV PLP ${ }^{\text {pro }}$ 与老鼠肝 炎病毒 $\mathrm{MHV}$, 牛冠状病毒 BoCoV, 禽支气管炎病毒 AIBV, 人冠状病毒 HCoV229E 以及传染性肠胃炎病 毒 TGEV 的 PLP pro 有 30\% 40\%的序列同源性. 以上 结果表明, PLP ${ }^{\text {pro }}$ 很有可能执行蛋白质水解功能.

$3 \mathrm{CL}^{\text {pro }}$ 是分子量为 $33.8 \mathrm{kD}$ 的酸性蛋白质, 含有 两个重复两次的肽段 TTTLN(24 28, 224 228 位置) 和 AGTD(173 176, 194 197 位置), TMHHMM 没有预 测到跨膜螺旋结构, BLIMPS 和 HMMPFAM 没有搜索 到相匹配的蛋白保守结构域, PSIBLAST 搜索发现 $3 \mathrm{CL}^{\text {pro }}$ 除了与 $\mathrm{MHV}, \mathrm{BoCoV}, \mathrm{AIBV}, \mathrm{HCoV}-229 \mathrm{E}, \mathrm{AIBV}$ 以及猪流行性痢疾病毒 PEDV 的 3CL ${ }^{\text {pro }}$ 有 40\% 50\% 的序列相似性以外, 还与蛋白质水解酶(Hydrolase)的 2879 3180 片段有 $43.8 \%$ 的相似性, 表明 $3 \mathrm{CL}^{\mathrm{pro}}$ 有水
解蛋白质的功能.

Rep 是分子量为 $106.5 \mathrm{kD}$ 的酸性蛋白质, TMAP 和 TMHMM 分析没有发现跨膜片段存在, HMMPFAM 搜索发现 Rep 与 HMM 数据库中的 PF00680 保守序列家族相匹配, PF00680 来自 RNA 依 赖的 RNA 聚合酶, PSIBLAST 搜索结果表明, SARS-CoV 的 Rep 与其他冠状病毒的 Rep 有 $60 \%$ 左 右的序列同源性. Hel 是分子量为 $66.9 \mathrm{kD}$ 的碱性蛋白 质, TMAP 和 TMHMM 没有预测到跨膜片段的存在, HMMPFAM 搜索发现 Hel 与 HMM 数据库中的 PF01443 (viral_helicase1 viral (superfamily 1) RNA helicase, 病毒螺旋酶 1 病毒(超家族)RNA 螺旋酶)保 守序列匹配, PSIBLAST 搜索结果表明, SARS-CoV 的 Hel 与其他冠状病毒的螺旋酶有 $60 \%$ 左右的同源性, 其中位于 282 288 的富含甘氨酶肽段 GPPGTGK 相当 保守，它形成一个位于 $\beta$-链和 $\alpha$-螺旋结构之间的柔性 环，该环能够与核苷酸的磷酸基团发生作用而参与 解螺旋, 被称为 P-loop ${ }^{[22]}$. 以上分析说明, 冠状病毒 的 $3 \mathrm{CL}^{\mathrm{pro}}$, Rep 和 Hel 具有很强的保守性.

$\mathrm{HD} 2 / \mathrm{NSP} 3$ 是分子量为 $33.0 \mathrm{kD}$ 的碱性蛋白质, TMHMM 分析表明该蛋白含有 7 个跨膜的 $\alpha$ 螺旋, $\mathrm{N}$ 末端 1 11 残基位于病毒粒子内部, C末端 236 290 残 基位于病毒外部, 其中 209 219 是一段富含半胱氨酸 的序列 CIMLVYCFLGYCCCCYFGLFC. PSIBLAST 搜索和序列比对结果表明, SARS-CoV HD2/NSP3 蛋 白与其他冠状病毒的序列同源性约在 $30 \%$ 左右. HMMPFAM 搜索发现, HD2/NSP3 的 43 140 肽段与 HMM 数据库的 PF00420(NADH-ubiquinone/plastoquinone oxidore, NADH-辅酶 $\mathrm{Q} /$ 泛醌氧化酶)保守序 列匹配, BLIMPS 搜索发现该蛋白的部分序列分别与 BLOCKS 数据库中的 IPB001169 (integrin beta, C-terminus, 整合 $\beta$ 蛋白 C-末端)、IPB002561 (filovirus glycoprotein，丝状病毒糖蛋白)、IPB000832(G-protein coupled receptors family, G-蛋白耦联受体家族)、 IPB002091(aromatic amino acids permease, 芳香族氨 基酸透性酶)保守序列相匹配, 暗示 HD2/NSP3 蛋白 很可能是位于病毒膜上的 $\mathrm{G}$-蛋白偶联受体家族成员, 具有信号传导功能.

NSP4 是一个分子量为 $9.3 \mathrm{kD}$ 的酸性蛋白质, 它 含有两段重复两次的序列 VLLS(12 15, 58 61 位置) 和 LLSVL(13 17, 55 59 位置). 与其他冠状病毒相比 较, NSP4 序列同源性约为 40\%左右. TMHMM 分析没 有发现跨膜螺旋, BLIMPS 和 HMMPFAM搜索没有发 
现任何匹配的保守序列家族. NSP5 是分子量为 21.9 $\mathrm{kD}$ 的中性蛋白, 没有预测到跨膜片段. HMMPFAM 分析发现, NSP5 的 16 117 肽段与 HMM 数据库的 PF04233 (phage Mu protein F like protein, 噬菌体 $\mathrm{Mu}$ 蛋白质类 $\mathrm{F}$ 蛋白) 保守序列匹配, 29 140 肽段与 PF04696(pinin/SDK/memA/protein conserved region, pinin/SDK/memA/蛋白保守区域)相匹配, BLIMPS 搜 索发现 NSP5 的部分序列分别与 BLOCKS 数据库中 的 IPB003660(HAMP domain, HAMP 结构域)、 PR00331 (haemagglutinin HA2 chain signature, 血球 凝集素 HA2 链信号)、PR00329 (haemagglutinin HA1/ HA2 chain signature, 血球凝集素 HA1/HA2 链信号) 保守序列模式匹配. 序列比对表明, SARS-CoV 的 NSP5 与其他冠状病毒的序列同源性约为 $40 \%$ 左右. NSP6 是分子量为 $12.4 \mathrm{kD}$ 的强碱性蛋白, TMAP 和 TMHMM 分析没有得到跨膜片段, BLIMPS 和 HMMPFAM 没有搜索到相匹配的保守序列模式, PSIBLAST 搜索和序列比对表明不同冠状病毒的 NSP6 蛋白序列同源性为 45\%左右.

生长因子样蛋白 GFLP (growth factor like protein) 是分子量为 $14.8 \mathrm{kD}$ 的酸性蛋白质, 不含跨膜区. BLIMPS 搜索发现该蛋白的 73 86 肽段与 BLOCKS 数据库中的 IPB000315(B-box zinc finger superfamily, B-盒锌指超家族) 保守序列模式匹配，36 74 肽段与 IPB003854(Gibberellin regulated protein, 赤霉素调节 蛋白)匹配. GFLP 在不同冠状病毒间的序列同源性约 为 $55 \%$ 左右.

蛋白质 NSP11，NSP12，NSP13 中都没有预测到 跨膜螺旋的存在. BLIMPS 搜索表明, NSP11 的 101 117 和 247 257 肽段分别与 BLOCKS 数据库中 的 PR00059 (ribosomal protein L6 signature, 核糖体 L6 蛋白质信号)和 IPB001608 (uncharacterized pyridoxal-5' -phosphate dependent enzyme family, 非典型 维生素 b6-5' - 依赖酶家族)保守序列模式相匹配, NSP13 的 179 215 肽段与 IPB000903 (myristoyl-CoA： protein N-myristoyltransferase, 肉豆冦酰-辅酶 A : 蛋 白质-肉豆冦酰转移酶) 匹配. HMMPFAM 搜索发现, NSP13 的 50 213 肽段与 HMM 数据库中的 PF01728 (FtsJ-like methyltransferase, 类-FtsJ 甲基移换酶)保守 结构域匹配. 没有在 HMM 和 BLOCKS 数据中找到 与 NSP12 匹配的保守蛋白序列. 不同冠状病毒的 NSP11 13 序列同源性分别约为 50\%, 40\%和 60\%左右.

以上结果表明, SARS-CoV ORF1ab 编码的蛋白
质产物与其他冠状病毒对应的蛋白质在序列上有很 强的保守性. BLIMPS, HMMPFAM 以及 PSIBLAST 搜索到的保守序列家族大多来自蛋白酶, 暗示 ORF $1 \mathrm{ab}$ 编码的蛋白质产物在病毒的生活周期中行使 各种各样的催化功能.

\subsection{SARS-CoV 结构蛋白质分析}

冠状病毒的 $\mathrm{S}$ 蛋白是一类较大的膜糖蛋白质, 大 部分氨基酸残基位于病毒粒子表面, 形成了病毒表 面的主要突起物, $S$ 蛋白三聚体使得病毒在电子显微 镜下呈现皇冠状形态. 多数冠状病毒的 $\mathrm{S}$ 蛋白在合成 后被水解成 $\mathrm{S} 1$ 和 $\mathrm{S} 2$ 两个亚基, 其中 $\mathrm{S} 1$ 亚基负责与 宿主细胞受体的识别和相互作用, S2 亚基负责病毒 与细胞膜融合, 因而, 冠状病毒的毒性强弱和宿主特 异性是由 $\mathrm{S}$ 蛋白决定的 ${ }^{[8]}$. SARS-CoV 的 $\mathrm{S}$ 蛋白由 1255 个氨基酸残基组成, 分子量为 $139.1 \mathrm{kD}$, 等电点 为 5.4, 是强酸性蛋白, 其 529 533，837 841 位置各 含有一个 FNGLT 重复片段. TMHMM 分析发现, S 蛋 白靠近 C 末端的 1196 1218 位置有一跨膜 $\alpha$ 螺旋, 而 $\mathrm{N}$ 末端 1 1195 区域位于病毒粒子表面, C末端 1219 1255 亲水区域位于病毒内部, 其中 1217 1236 (CCMTSCCSCLKGACSCGSCC) 为富含半胱氨酸的肽段. HMMPFAM 搜索发现, SARS-CoV 的 75 609 区域与 HMM 数据库中冠状病毒 S1 结构域 PF01600 保守序 列匹配, 641 1247 区域与 PF016001(冠状病毒 S2 结构 域)保守序列相匹配. 其他冠状病毒 $S$ 蛋白剪切位点 的序列特征通常为 RRXRR, RXRR, 但是对于 SARS-CoV, 没有搜索到这样的碱性剪切位点, 因此, SARS-CoV 的 S 蛋白很有可能是以 $\mathrm{S} 1$ 和 S2 两个结构 域形式而不是以 $\mathrm{S} 1$ 和 $\mathrm{S} 2$ 亚基形式存在. 通过 PSIBLAST 搜索其他冠状病毒 S 蛋白序列以及 CLUSTALW 序列比对, 我们发现 SARS-CoV 的 S2 较 S1 在序列上 更为保守, 前者与其他冠状病毒的序列同源性为 18\% 21\%, 后者为 34\% 35\%, 这一结果能够很好地 解释 $\mathrm{S}$ 蛋白的功能: $\mathrm{S} 1$ 负责与特异性受体的识别和结 合, 由于不同冠状病毒侵染不同的类型的宿主细胞, 因此 S1 亚基具有多样性; 而 S2 亚基负责病毒膜与细 胞膜的融合, 这种功能上的单一性使得不同冠状病 毒的 S2 亚基相对保守. 目前, 冠状病毒的侵染机制 可以用坞塌模型(collapse model)来解释: 首先, S1 亚 基通过与宿主细胞膜上的特异性受体作用而发生构 象变化, 这种构象变化能够诱导 S2 亚基发生结构改 变, 具体地讲, S2 C 端的多个双亲性 $\alpha$ 螺旋坞塌成 
coiled-coil 构象, 使得 S2 的融合肽段靠近并插人细胞 膜, 导致膜融合 ${ }^{[8]}$. 我们用 Workbench 生物信息学平 台提供的 8 种蛋白质二级结构预测方法对 $\mathrm{S}$ 蛋白质进 行了二级结构预测, 发现 750 1010 区域能够形成 9 个连续的 $\alpha$ 螺旋结构(图 1), 进一步用 GREASE 方 法 ${ }^{[23]}$ 分析 $\mathrm{S}$ 蛋白的亲疏水特性, 发现 885 950, 973 990 区域呈现明显的亲水性和疏水性交替出现 的性质, 说明这部分区域很可能是发生构象坍塌的 双亲性 $\alpha$ 螺旋区域. 最近的研究指出, HCoV-229E S 蛋白的 417 547 氨基酸区域是病毒结合人类细胞膜 受体氨肽酶 $\mathrm{N}$ 所必须的 ${ }^{[24]}$, 我们将 SARS-CoV S 蛋白 与 $229 \mathrm{E}$ 的 S 蛋白进行序列比对, 发现 SARS-CoV S 蛋白的 492 651 区域与 229E 的 417 547 区域相对应, 但是, $S$ 蛋白的 492 651 区域是否是 SARS 病毒结合 受体所必须的, 需要进一步的实验验证.

SARS-CoV E 蛋白是包含 76 个氨基酸的小酸性 蛋白质, 分子量为 $8.3 \mathrm{kD}$. TMAP 和 TMHMM 分析发 现 E 蛋白的 12 34 位置存在一疏水跨膜螺旋区域, 残 基 1 11 位于病毒内部, 而 35 76 亲水肽段位于病毒 表面. HMMPFAM 搜索发现 $\mathrm{E}$ 蛋白整条序列与 HMM 数据库中的 PF02723(NS3/small envelope protein E, NS3/小包被蛋白质 E) 保守序列相匹配, BLIMPS 搜索 也发现 E 蛋白的 36 67 区域与 BLOCKS 数据库中的 冠状病毒小包被蛋白序列家族 IPB003873 相匹配. 但 是, PSIBLAST 搜索只找到两种冠状病毒具有与 HCoV-E 蛋白同源的蛋白序列, 它们是鼠肝炎病毒 MHV 和鼠腮腺炎冠状病毒 RSCoV (rat sialodacryoadenitis coronavirus), 与 SARS-CoV E 蛋白的序 列同源性分别为 $23 \%$ 和 $24 \%$, 说明 $\mathrm{E}$ 蛋白在冠状病毒 进化中较为不保守. 此外有研究发现, 猪肠胃炎病毒 TGEV 的 E 蛋白对病毒复制起关键作用 ${ }^{[25]}$, 但是在鼠 肝炎病毒 MHV 中, $\mathrm{E}$ 蛋白基因的删除只能使病毒复 制效率降低 ${ }^{[26]}$. SARS-CoV 的 E 蛋白除了参与病毒外 壳形成外是否对病毒复制起作用, 需要进一步研究 才能确定.

M 蛋白质是包含 221 个氨基酸的碱性蛋白, 分子 量为 $25.1 \mathrm{kD}$. 其 $\mathrm{N}$ 端 44 97 氨基酸组成一强疏水区 域, 21 24 以及 137 140 位置各包含一段重复序列 LVIG. PSIBLAST 搜索和序列比对发现, SARS-CoV 109 114 位置存在相当保守的一段序列 WSFNPE，而 不同冠状病毒 $\mathrm{M}$ 蛋白整体上的序列同源性仅 $30 \%$ 左 右. TMHMM 方法预测到 3 个跨膜螺旋片段: $15 \sim 37$,
50 72, 77 99, N 端 1 14 和 C 端 100 221 肽段分别位 于病毒粒子外部和内部. 位于病毒内部的肽段含有 丰富的正电氨基酸, 推测能够与 $\mathrm{N}$ 蛋白或病毒 RNA 发生相互作用. HMMPFAM 和 BLIMPS 搜索均发现, SARS-CoV M 蛋白全序列与冠状病毒 M 糖蛋白 (Coronavirus M matrix/glycoprotein, PF01635, IPB002574)保守序列家族相匹配, 说明 SARS-CoV M 蛋白 是比较典型的冠状病毒 $M$ 蛋白. 此外, BLIMPS 搜索 还发现, M 蛋白的部分序列与 BLOCKS 数据库中的 IPB001717 (anion exchanger family, 阴离子交换器家 族)、IPB001425 (bacterial rhodopsin, 细菌视紫红质)、 IPB001712 (bacterial export FHIPEP family, 细菌输出 FHIPEP 家族)相匹配, 暗示 $\mathrm{M}$ 蛋白可能与离子交换 和信号传导有关. 此外, 对其他冠状病毒的研究表明, $\mathrm{S}$ 蛋白与 $\mathrm{M}$ 蛋白的相互作用是病毒外壳蛋白积累和 包装的关键步骤 ${ }^{[10]}, \mathrm{SARS}-\mathrm{CoV} \mathrm{S}$ 蛋白的等电点 $\mathrm{pI}$ 为 $5.4, \mathrm{M}$ 蛋白为 9.8 , 可以推测, 二者之间的静电相互 作用对病毒装配起一定的作用.

$\mathrm{N}$ 蛋白是包含 422 个氨基酸的强碱性蛋白, 分子 量为 $46.0 \mathrm{kD}$, 其 191 195 和 203 207 位置各有一段 重复序列 SRGNS. TMAP 和 TMHMM 方法都没有预 测到 $\mathrm{N}$ 蛋白中有跨膜螺旋的存在. 通过 PSIBLAST 搜索和序列比对，我们发现 N 蛋白的 107 118 位置有 一段相当保守的序列 PRWYFYYLGTGP, 目前还不 清楚此保守序列的功能. 此外, SARS-CoV N 蛋白的 373 390 位置有一段富含赖氨酸的序列 KKKKTDEAQPLPQRQKKQ, 该序列与 PFAM 数据库中的双向核 酸定位信号(bipartite nuclear targeting signal) QDOC50079 相匹配, 负责核酸的识别和结合. $\mathrm{N}$ 蛋白 的 177 207 位置还有一段富含丝氨酸的亲水氨基酸 序列 SRGGSQASSRSSSRSRGNSRNSTPGSSRGNS, 该序列是否与核酸作用还不清楚. BLIMPS 搜索发现 $\mathrm{N}$ 蛋白除了与 BLOCKS 数据库中的 IPB001218 (Coronavirus nucleocapsid protein, 冠状病毒核壳体 蛋白)匹配外, 部分序列还分别与 IPB002565 (Orbivirus NS3, 环状病毒 NS3)、IPB000096 (serum amyloid A protein, 血清淀粉样 A 蛋白)、IPB001677 (transferrin binding protein, 转铁蛋白结合蛋白)、 IPB000689 (UbiH/COQ6 monooxygenase family, UbiH/ COQ6 单加氧酶家族)等序列家族相匹配. 不同冠状 病毒的 $\mathrm{N}$ 蛋白保守性不是很强, 序列同源性约为 $30 \%$ 左右.

\section{3 功能未知蛋白分析}

由 SARS-CoV 基因组推测的 X1 蛋白质是一个包 


SARS S protein
BPS
D_R
DSC
GGR
GOR
H_K
K_S
JOI

SARS S protein
BPS
D_R
DSSC
GGR
GOR
H_K
K_S
JOI

SARS S protein
BPS
D_R
DSC
GGR
GOR
H_K
K_S
JOI

SARS S protein
BPS
D_R
DSC
GGR
GOR
H_K
KES
JOI

SARS S protein BPS

D_R

$\mathrm{D} \overline{\mathrm{S} C}$

GGR

GOR

H_K

$\mathrm{K}$ S $\mathrm{J} \overline{\mathrm{O}}$ $\begin{array}{lrrrr}740 & 750 & 760 & 770 & 780\end{array}$

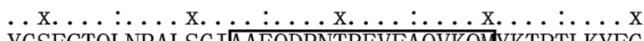
YGSFCTQLNRALSGI AAEQDRNTREVFAQVKQMYKTPTLKYFG EEEEEECHHCCCCCOCCHHHHHEHHCCCCCCHHECCCCCCCCC CCCEЕССССНHСССЕ НHНHССССЕНЕЕНЕЕЕЕЕССССССССЕС ССССССНHНHНHНHННHНHНHНHНHНHНHНHН ОССССЕЕЕЕС СССССНННННННННН НННННССНHННННННННН СССССЕЕЕСС CCCCEЕЕЕСССССССССННСССННННННННННН СССССЕЕЕЕС ССССССССССССССН НHНHНHНHНHНHНHНHНН ОСССССЕЕЕС ССССССССССССССОССССССССССНННННHНH НССССССССС СССССССССССССССНHНHНССНHНHНHНHНHН ССССССЕЕЕ

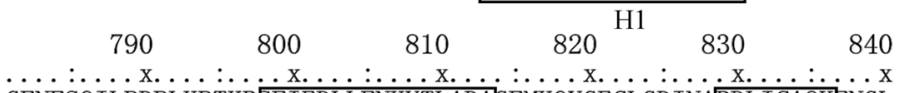

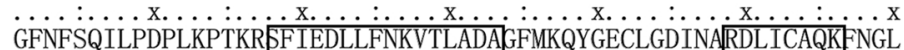
СССССССССССССССНHСНССННННННССНННН СССССССССССССНН ННННССНHЕССС CCСЕЕСЕССССССССССЕЕЕНHЕНЕЕЕЕЕЕНСН ССНССССССССССС ССЕЕЕНHС СССЕ

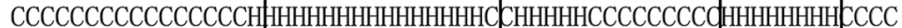

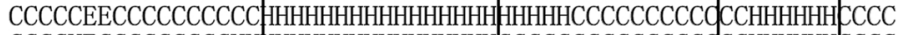

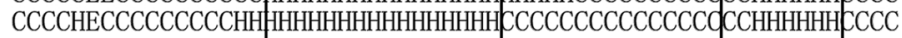
СССССССССССССССССССННННННННННСССС ССССССССССССННННННННННССССС СCССССССССССССССС ССС ССССССССССССССССС НHНHНHНHНHНHHHHH ССССССССССССССОССНHНHНHСССС

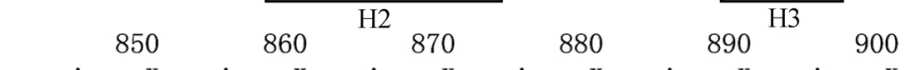

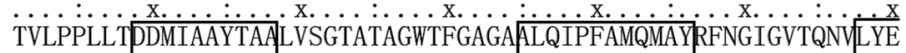
СССССССС НННННННННН СССССССССССССССС ССССССССНННННССССССЕЕЕЕННН

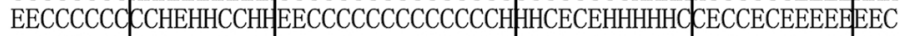
СССССССС НHНHНHНHЕЕ ЕЕЕССССЕЕЕЕЕССССССССНHНHНHНHЕЕСССССЕЕЕННHН

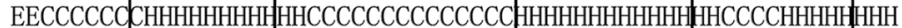

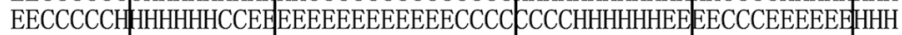

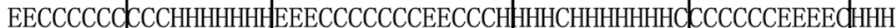
ССССЕЕСС НHНHНHНHНH ЕССССССССССССССС СССССССССССОССССССССССОССС ЕЕССССССНHНHНHНHНH ЕЕССССССССССССССССССНHНHНHНССССССССЕЕЕСНHН

$910^{\mathrm{H} 4} 920 \quad 930 \quad 940^{\mathrm{H} 5} \quad 950 \quad 960$

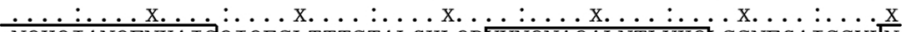
NQKQIANQFNKAISQIQESLTTTSTALGKLQD VVNQNAQALNTLVKQLSSNFGAISSVIIN

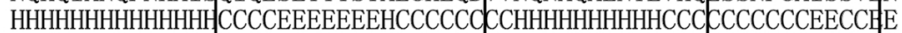
ССССЕНСССССНЕЕ ЕЕСНСЕСССССССССЕСС ЕЕСССНHНHСЕЕЕЕЕЕССССССЕЕЕЕВС НHНHНHНHНHНHНH НЕЕСССССССССССНHН ННHНHНHНHНHНHНH НСССССЕЕЕЕННН НННННННННННННН ННННСССССННННССННН НННННННННННННННН НСССССССННННН ННННННННННССНН ЕЕЕЕЕЕЕЕСННННСЕЕЕЕ ННННННННННННННЕССССССЕЕЕЕЕНН HHHHHHHНCCCCC EEEEECCCEECCCCCCCCOCCCCCHHHHHEEEEECCCCCCCCEEECH СССССССССССННОНССССССССССССССССС СССССССНННННННН ССССССССССССС

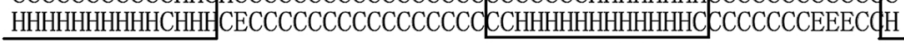
$\mathrm{H} 6$

$\begin{array}{llllll}970 & 980 & 990 & 1000^{\mathrm{H} 7} & 1010 & 1020\end{array}$

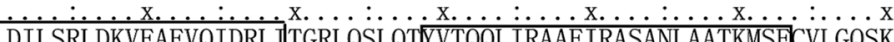
HCCCCCCCCCCCCCCEC CCCHHECCE EEEHHHHHHHHHHHHHHHHHHHHH CCCCCCC

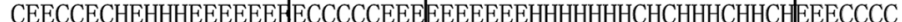

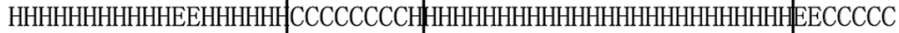
ННННННННННННННННННН НСССССННН ННННННННННННННННННННННННН ННССССС

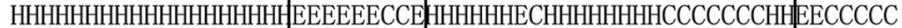

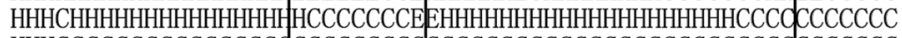
НHНССССССССССССССС ССССССССОССССССССССССССССССССССССО СССССС

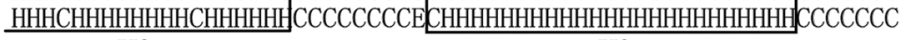
$\mathrm{H} 8$

H9

图 18 种二级结构预测方法预测出的 $\mathrm{S}$ 蛋白二级结构

方框表示 3 种以上预测方法预测出的 $\alpha$ 螺旋区域 H1 H9
含 274 个氨基酸的酸性蛋白质, 分子量为 $30.9 \mathrm{kD}$. 序 列分析发现 79 115 位置有一强疏水片段 FICNLLLLFVTIYSHLLLVAAGMEAQFLYLYALIYFL. TMAP 和 TMHMM 预测到 3 个跨膜的螺旋区域: 34 56, 77 99, 103 125, $\mathrm{N}$ 末端 1 33 残基位于病毒粒子外部, 而 C 末端 126 274 肽段位于病毒内部. PSIBLAST 没 有搜索到与 X1 同源的蛋白质序列, HMMPFAM 没有
搜索到与 X1 相匹配的任何保守序列家族. BLIMPS 搜索发现, X1 的 231 252 肽段与 BLOCKS 数据库中 的 PR01542 (foot-and-mouth disease virus VP1 coat protein signature, 口足疾病病毒 VP1 外壳蛋白信号) 保守序列匹配，67 87 肽段与 PR00699 (C. elegans integral membrane protein Srb signature, 线虫整合膜 蛋白 Srb 信号)保守序列匹配, 暗示 X1 可能是存在于 
SARS-CoV 蛋白质外壳上的结构蛋白.

X2 是包含 154 个氨基酸残基的强碱性蛋白质, 其正电氨基酸赖氨酸 + 精氨酸含量与负电氨基酸精 氨酸 + 甘氨酸含量的比例为 $22: 1 . \mathrm{X} 2$ 蛋白序列的 28 31 以及 95 98 位置各有一小段重复序列 SLLK. PSIBLAST 没有搜索到与 X2 同源的其他冠状病毒蛋 白序列. C 末端 137 154 KKVSTNLCTHSFRKKQVR 序列与 PFAM 数据库中的双向核定位信号 PS50079|NLS_BP (bipartite nuclear localization signal) 相匹配. TMAP 和 TMHMM 分析没有预测到任何跨膜 螺旋片段的存在. 以上结果暗示 X2 蛋白很可能与 $\mathrm{N}$ 蛋白相似(详见对 $\mathrm{N}$ 蛋白的讨论), 位于病毒粒子内部, 行使与 RNA 结合的功能, 同时 X2 蛋白在病毒包装过 程中可能起关键作用.

X3 蛋白是包含 63 个氨基酸的小酸性蛋白质, 分 子量为 $7.5 \mathrm{kD}$. PSIBLAST 搜索没有找到任何该蛋白 的同源序列, HMMPFAM 和 BLIMPS 分析也没有发现 任何匹配的保守序列家族. TMHMM 没有预测到跨膜 $\alpha$ 螺旋, 但是 TMAP 分析发现 9 37 位置可能存在一 段跨膜片段, $\mathrm{N}$ 末端 1 8 区域位于病毒外部, 根据以 上信息不足以推测 X3 蛋白的功能. X4 蛋白是包含 122 个氨基酸的碱性蛋白, 分子量为 $13.9 \mathrm{kD}$. 其 101 115 位置有一强疏水片段 LFLIVAALVFLILCF, TMAP 和 TMHMM 同时预测到 X4 的 95 117 位置存 在一个跨膜 $\alpha$ 螺旋结构, $\mathrm{N}$ 末端 1 94 区域位于病毒粒 子外部, 而 C末端 118 122 肽段位于病毒内部, 我们 还注意到 $\mathrm{C}$ 末端尾巴 118 120 位置有 3 个连续的碱 性氨基酸 KRK, 推测它们很可能与核酸发生作用. BLIMPS 搜索发现 X4 蛋白的 101 119 序列与 BLOCKS 数据库的四环素抗性蛋白 TetB 信号 PR01036 (tetracycline resistance protein TetB signature, 四环素抗性蛋白 TetB 信号)序列家族匹配, 而 PSIBLAST 搜索没有发现 X4 的同源蛋白序列. 以上 分析表明, X4 蛋白很可能是位于病毒膜上的结构蛋 白, 对病毒粒子的装配可能起一定的作用. X5 蛋白是 包含 84 个氨基酸的碱性蛋白质, 分子量为 $9.5 \mathrm{kD}$, PSIBLAST 没有搜索到其他冠状病毒的同源蛋白序 列. TMAP 和 TMHMM 没有预测到跨膜片段. BLIMPS 搜索发现 X5 的 52 68 序列与 BLOCKS 数据库中的 IPB000001(kringle, 一种环状结构蛋白)保守序列家 族匹配. 这些分析表明, X5 蛋白质很可能位于病毒粒 子内部, 其 $\mathrm{N}$ 末端和 $\mathrm{C}$ 末端的强正电区域可能会与
核酸或 $\mathrm{M}$ 蛋白发生作用, 促进病毒装配.

HP5 是分子量为 $10.8 \mathrm{kD}$ 的强酸性蛋白质, 它包 含 98 个氨基酸残基. TMAP 和 TMHMM 没有预测到 跨膜片段. HMMPFAM 和 BLIMPS 没有搜索到与该蛋 白匹配的保守序列模式, PSIBLAST 搜索没有发现任 何同源蛋白质序列. 根据以上信息不足以推测 HP5 的功能.

可读框 ORF9 编码一个包含 44 个氨基酸的小酸 性蛋白质, 分子量为 $5.3 \mathrm{kD}$. 负电氨基酸全部集中在 蛋白质的 $\mathrm{N}$ 末端和 C末端. PSIBLAST 没有搜索到任 何同源序列. TMAP 和 TMHMM 预测 9 31 位置存在 一段跨膜 $\alpha$ 螺旋, $\mathrm{N}$ 端 1 8 肽段位于病毒内部, 而 $\mathrm{C}$ 端 32 44 区域位于病毒表面. BLIMPS 搜索发现 ORF9 的 11 32 序列与 BLOCKS 数据库中的 PR00697(C. elegans Sra family integral membrane protein signature, 秀丽隐杆线虫 C. elegans Sra 整合膜蛋白信号)保守序 列家族匹配，8 32 肽段与 IPB001898 (sodium:sulfate symporter family, 钠: 硫酸盐同向转移蛋白质家族)匹 配, 还有部分肽段分别与 IPB003804(L-lactate permease, L-乳酸盐透性酶)、PR01434 (NADH-ubiquinone oxidoreductase chain 5 signature, NADH-辅酶 $\mathrm{Q}$ 氧化还原酶 链 5 信号)、PR01535(vomeronasal type 2 receptor family signature, 鼻犁骨器类型 2 受体家族信号)、IPB001421 (mitochondrial ATPase subunit 8, 线粒体 ATP 酶亚基 8)、IPB003362 (bacterial sugar transferase, 细菌糖转 移酶)、PR00169 (potassium channel signature, 钾离子 通道信号)、PR00701(60 kD inner membrane protein signature, $60 \mathrm{kD}$ 内部膜蛋白信号)等保守序列家族相 匹配，从这些结果可以推测 ORF9 编码的蛋白质很可 能是位于病毒膜上的结构蛋白, 其功能或许与离子 转运有关. 可读框 ORF10 编码一个仅包含 39 个氨基 酸的碱性蛋白质, 分子量为 $4.3 \mathrm{kD}$. PSIBLAST 没有 搜索到任何该蛋白质的同源序列. TMHMM 没有预测 到跨膜螺旋的存在, 但是 TMAP 预测出 3 31 位置有 一跨膜区域. BLIMPS 搜索发现该蛋白的 4 29 序列与 BLOCKS 数据库中的 IPB001010(富硫蛋白 Thionin) 相匹配, 2 25 与 6 15 序列分别与 PR01520(zeta- tubulin signature, zeta-微管蛋白信号)、IPB003606 (N-terminal to some SET domains, N-末端至 SET 结构域)保守序 列匹配. ORF14 编码一个包含 70 个氨基酸的分子量 为 $7.8 \mathrm{kD}$ 酸性蛋白质. PSIBLAST 没有搜索到该蛋白 质的任何同源序列. TMHMM 没有预测到跨膜螺旋的 1 存在, 但是 TMAP 预测结果表明在 34 61 位置存在一 
个跨膜片段. HMMPFAM 和 BLIMPS 搜索没有找到与 该蛋白匹配的任何保守序列家族. 无法从以上信息 推测 ORF10 和 ORF14 编码的蛋白质的功能.

\subsection{SARS-CoV 蛋白质进化地位分析}

我们用 PSIBLAST 程序对 SARS-CoV 的每一个 蛋白质序列在非冗长蛋白质序列数据库中进行同源 序列搜索, 对能搜索到同源序列的蛋白质进行进化 距离分析(表 3), 并绘制无根进化树(数据未列出), 以 确定 SARS-CoV 的分类地位以及与其他冠状病毒的 亲缘关系。

进化树分析显示, 所有能搜索到同源序列的 SARS-CoV 蛋白质并不归属于以前划分的任何一组 冠状病毒, 它们无一例外地区别于其他 3 组冠状病毒 对应的蛋白而形成一个独立的分支, 这一结果与文 献 ${ }^{[5,6]}$ 的报道是一致的. 同时, 进化距离矩阵计算表 明, 除 PLP 和 $\mathrm{N}$ 蛋白质之外, SARS-CoV 的大多数蛋 白质与其他每种冠状病毒对应的蛋白质在进化距离 上近乎相等, 例如, SARS-3CL ${ }^{\text {pro }}$ 与其他冠状病毒的 进化距离在 2.61 3.52 范围内变动, 平均距离为 3.09, 标准误差为 0.36 ; Rep 在 1.40 1.84 范围内变动, 平均 距离为 1.66 , 标准误差为 $0.21 ; \mathrm{Hel}$ 在 $1.26 \sim 1.78$ 范围 内变动, 平均距离为 1.52 , 标准误差为 0.22 等等(表 3 ). 以上分析支持了文献 ${ }^{[5,6]}$ 的结果, 即 SARS-CoV 病 毒不属于以前划分的其他 3 组冠状病毒而应该属于 第 4 组. 尽管如此, 进化距离分析表明 SARS-CoV 的 Hel 和 Rep 与其他冠状病毒的对应蛋白质具有很近的 亲缘关系，它们之间的平均进化距离小于 1.7(表 3).
Hel 和 Rep 负责病毒 RNA 的复制和转录, 功能上的 保守性使得它们承受较大的进化压力, 因而蛋白质 的序列和结构显得更为保守. 此外, 通过比较不同冠 状病毒蛋白质间的进化距离, 我们还发现一个有趣 的信息, 即牛冠状病毒 BoCoV 和鼠肝炎病毒 MHV 的 大部分蛋白质在亲缘关系上更靠近 SARS-CoV 冠状 病毒, 例如 BoCoV 有 7 个蛋白质与 SARS-CoV 的对 应蛋白质在进化距离最为接近, 它们是 PLP, NSP4, Rep, Hel, NSP11, NSP12 和 S 蛋白, MHV 有 6 个蛋白 质与 SARS-CoV 的对应蛋白质在进化距离上最接近, 它们是 $3 \mathrm{CL}^{\mathrm{pro}}, \mathrm{HD} 2, \mathrm{GFLP}, \mathrm{Rep}, \mathrm{M}$ 和 $\mathrm{N}$ 蛋白. 但是, 目前还没有证据表明 SARS-CoV 的蛋白质编码序列是 由牛、鼠或其他物种的冠状病毒的对应序列重组而来.

\section{3 结论}

SARS-CoV 相关蛋白质的进化树和进化距离分 析表明，该病毒是一种全新的冠状病毒，它既不是由 已知的冠状病毒重组而来, 也不是从已知的人或其 他动物的冠状病毒突变而来. 我们推测其来源有两 种途径: 一种可能是由未知动物冠状病毒突变或重 组而来, 同时获得了感染人体细胞的能力和致病能 力; 另一种可能是由未知的无毒害的人类冠状病毒 突变而来，但是没有在未被 SARS-CoV 病毒感染的 人体内分离出该病毒的相关抗体 ${ }^{[3]}$, 表明这种未知的 “温和”病毒可能是不存在的. 建议用 SARS 病人的血 清在第 1 例 SARS 病例发现地对野生和家养动物做大 规模抗原-抗体反应, 以查出 SARS-CoV 的动物宿主 或“源头”. 本文即将完成时, 香港大学一研究小组宣

表 3 SARS-CoV 相关蛋白质与其他冠状病毒对应蛋白质的进化距离比较 ${ }^{\text {a) }}$

\begin{tabular}{|c|c|c|c|c|c|c|c|c|c|c|c|c|c|c|c|c|c|}
\hline $\begin{array}{c}\text { SARS- } \\
\text { CoV }\end{array}$ & MHV & $\mathrm{BoCoV}$ & PEDV & $\begin{array}{c}\mathrm{HCoV}- \\
229 \mathrm{E}\end{array}$ & TGEV & AIBV & FIPV & PRCoV & $\mathrm{CCoV}$ & RSCoV & $\begin{array}{c}\mathrm{HCoV}- \\
\mathrm{OC} 43\end{array}$ & PHEV & TuCoV & ECoV & $\mathrm{PuV}$ & $\begin{array}{l}\text { 平均进 } \\
\text { 化距离 }\end{array}$ & $\begin{array}{l}\text { 标准 } \\
\text { 误差 }\end{array}$ \\
\hline PLP & 7.22 & 7.09 & 9.26 & 9.49 & 9.86 & 9.84 & & & & & & & & & & 8.79 & 1.29 \\
\hline $3 \mathrm{CL}^{\mathrm{pro}}$ & 2.61 & 2.67 & 2.99 & 3.52 & 3.17 & 3.51 & 3.17 & & & & & & & & & 3.09 & 0.36 \\
\hline HD2 & 5.81 & 6.05 & 6.34 & 7.15 & 6.90 & 8.41 & & & & & & & & & & 6.78 & 0.95 \\
\hline NSP4 & 2.73 & 2.54 & 3.42 & 3.53 & 2.94 & 3.05 & & & & & & & & & & 3.04 & 0.38 \\
\hline NSP5 & 2.56 & 2.64 & 2.59 & 2.22 & 2.50 & 3.09 & & & & & & & & & & 2.60 & 0.28 \\
\hline NSP6 & 3.44 & 2.96 & 2.85 & 3.09 & 3.43 & 3.41 & & & & & & & & & & 3.20 & 0.26 \\
\hline GFLP & 2.14 & 2.25 & 2.18 & 2.69 & 2.49 & 2.16 & & & & & & & & & & 2.31 & 0.22 \\
\hline Rep & 1.40 & 1.40 & 1.78 & 1.84 & 1.82 & 1.74 & & & & & & & & & & 1.66 & 0.21 \\
\hline $\mathrm{Hel}$ & 1.26 & 1.22 & 1.60 & 1.65 & 1.60 & 1.78 & & & & & & & & & & 1.52 & 0.22 \\
\hline NSP11 & 1.71 & 1.70 & 2.24 & 2.20 & 2.21 & 2.24 & & & & & & & & & & 2.05 & 0.27 \\
\hline NSP12 & 2.53 & 2.49 & 2.81 & 3.16 & 2.99 & 3.32 & & 12.18 & & & & & & & & $2.88^{*}$ & 0.34 \\
\hline S & 5.63 & 5.33 & 8.06 & 6.62 & 7.53 & 7.19 & 7.11 & 7.56 & 7.31 & 5.65 & 5.4 & 5.60 & & & & 6.58 & 0.99 \\
\hline M & 3.35 & 3.37 & 4.59 & 5.13 & 4.90 & 5.85 & 4.93 & & 4.93 & 3.54 & & 3.36 & 16.43 & & & $4.40^{*}$ & 0.91 \\
\hline $\mathrm{N}$ & 4.02 & 4.05 & 7.13 & 6.14 & 6.27 & 5.71 & 6.14 & & 6.18 & 4.07 & 4.13 & 4.08 & 5.69 & 4.03 & 4.08 & 5.12 & 1.44 \\
\hline
\end{tabular}

a) MHV一一鼠肝炎病毒, BoCoV一一牛冠状病毒, PEDV一一猪流行性痢疾病毒, HCoV-229E一人冠状病毒 229E, TGEV一一传染性肠胃炎病毒, AIBV一一禽传染性支气管炎病毒, FIPV一一猫传染性腹膜炎病毒 PRCoV一一猪呼吸道冠状病毒, CCoV一一大冠状病毒, RSCoV一一鼠唾液腺冠状病毒,

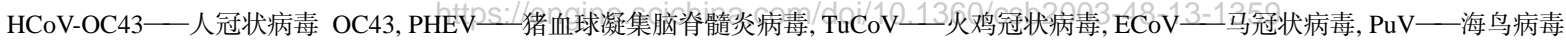


布他们在深圳一家市场的 6 只果子猖体内分离出了 类似于 SARS-CoV 的冠状病毒, 同时, 该市场 10 名 与果子狸长期接触的人员当中有 5 人体内分离出了 SARS 病毒抗体 ${ }^{[27]}$, 但是, 果子狸的冠状病毒是如何 获得感染人体细胞能力的? 突变还是重组? 果子狸 冠状病毒演变成 SARS-CoV 后是否还具有侵染果子 狸的能力? 是否会导致果子狸的下呼吸道感染? 是 否还存在 SARS-CoV 的其他动物宿主? 这些问题的 解决对于能否彻底消灭 SARS 是至关重要的.

我们对 SARS-CoV 所有蛋白质的分子量、等电 点、分子消光系数的预测对于这些蛋白质的分离、提 取和纯化提供了有用的信息, 同时, 蛋白质的跨膜区 预测、保守序列家族搜索以及同源序列比对对于预测 功能未知蛋白的功能提供了有用的信息. 我们的分 析结果表明, ORF1ab 编码的蛋白质产物主要与多蛋 白质体的剪切和病毒 RNA 的复制与转录有关; X1 蛋 白具有跨膜螺旋结构的同时还具有与膜蛋白 Srb 信 号保守序列家族相似的氨基酸序列, 表明 X1 可能是 存在于 SARS-CoV 蛋白质外壳上的结构蛋白; X2 蛋 白不具有跨膜结构, 但是有一段双向核定位信号序 列, 同时它为强碱性蛋白, 表明 X2 很可能与 RNA 结 合, 对病毒包装起作用; $S$ 蛋白的二级结构特征和亲 疏水性特征能够很好地支持冠状病毒的坍塌侵染模 型, 同时, 我们对 $\mathrm{S}$ 蛋白的生物信息学分析为寻找针 对 $\mathrm{S}$ 蛋白的抗原位点提供了信息. 我们的工作为理论 构建 SARS-CoV 相关蛋白质的结构以及实验测定 SARS-CoV 蛋白质组奠定了基础, 从长远的观点看, 还为抗 SARS 药物设计以及 SARS 疫苗研制提供了一 些有用的信息.

\section{参考文献}

1 Peiris J S M, Lai S T, Poon L L M. Coronavirus as a possible cause of severe acute respiratory syndrome. Lancet, 2003, 361: 1319 1325

2 Drosten C, Günther S, Preiser W, et al. Identification of a novel coronavirus in patients with severe acute respiratory syndrome. $\mathrm{N}$ Engl J Med, 2003, 348: 1967 1976

3 Ksiazek T G, Erdman D, Goldsmith C S, et al. A novel coronavirus associated with severe acute respiratory syndrome. N Engl J Med, 2003, 348: 1947 1958

4 秦鄂德, 祝庆余, 于曼, 等. SARS 相关病毒(BJ01 株)的全序列 及其比较分析. 科学通报, 2003, 48(11): 1127 1134

5 Rota P A, Oberste M S, Monroe S S, et al. Characterization of a novel coronavirus associated with severe acute respiratory syndrome. Science, 2003, 300:1394 1399

6 Marra M A, Jones S J, Astell C R, et al. The genome sequence of the SARS-associated coronavirus. Science, 2003, 300: 1399 1404

7 Jun R Y, Lin W C, Ling A E, et al. Comparative full-length ge- nome sequence analysis of 14 SARS coronavirus isolateds and common mutations associated with putative origins of infection. Lancet, 361: 1779 1790

8 Gallagher T M, Buchmeier M J. Coronavirus spike protein in viral entry and pathogenesis. Virology, 2001, 279: 371 374

9 Bonavia A, Zelus B D, Wentworth D E, et al. Identification of a receptor binding domain of the spike glycoprotein of human coronavirus HCoV-229E. J Virol, 2003, 77: 2530 2538

10 Garoff H, Hewson R, Opstelten D J. Virus maturation by budding. Microbiol Mol Biol Rev, 1998, 62: 1171 1190

11 Boeckmann B, Bairoch A, Apweiler R, et al. The Swiss-Prot protein knowledgebase and its supplement TrEMBL in 2003. Nucleic Acids Res, 2003, 31: 365 370

12 Herold J, Raabe T, Schelle-Prinz B, et al. Nucleotide sequence of the human coronavirus 229E RNA polymerase locus. Virology, 1993, 195: 680 691

13 Brendel V, Bucher P, Nourbakhsh I, et al. Methods and algorithms for statistical analysis of protein sequences. Proc Natl Acad Sci USA, 1992, 89: 2002 2006

14 Subramaniam S. The Biology workbench-A seamless database and analysis environment for the biologist [editorial]. Proteins, 1998, 32: 1 2

15 Persson B, Argos P. Prediction of transmembrane segments in proteins utilising multiple sequence alignments. J Mol Biol, 1994, 237: $182 \sim 192$

16 Sonnhammer E L, Heijne G Von, Krogh A. A hidden Markov model for predicting transmembrane helices in protein sequences. Proc Int Conf Intell Syst Mol Biol, 1998, 6: 175 182

17 Bateman A, Birney E, Cerruti L, et al. The Pfam protein families database. Nucleic Acids Res, 2002, 30: 276 280

18 Wallace J C, Henikoff S. PATMAT: A searching and extraction program for sequence, pattern and block queries and databases. Comput Appl Biosci, 1992, 8: 249 254

19 Altschul S F, Madden T L, Schaffer A A. Gapped BLAST and PSI-BLAST: a new generation of protein database search programs. Nucleic Acids Res, 1997, 25: 3389 3402

20 Thompson J D, Higgins D G, Gibson T J. CLUSTALW: improving the sensitivity of progressive multiple sequence alignment through sequence weighting, position-specific gap penalties and weight matrix choice. Nucleic Acids Res, 1994, 22: 4673 4680

21 Felsenstein J. PHYLIP-Phylogeny Inference Package (Version 3.2). Cladistics, 1989, 5: 164 166

22 Saraste M, Sibbald P R, Wittinghofer A. The P-loop-a common motif in ATP- and GTP-binding proteins. Trends Biochem Sci, 1990, 15: 430 434

23 Kyte J, Doolittle R F. A simple method for displaying the hydropathic character of a protein. J Mol Biol, 1982, 157: 105 132

24 Bonavia A, Zelus B D, Wentworth D E, et al, Identification of a receptor-binding domain of the spike glycoprotein of human coronavirus HCoV-229E. J Virol, 2003, 77: 2530 2538

25 Ortego J, Escors D, Laude H, et al. Generation of a replication-competent, propagation-deficient virus vector based on the transmissible gastroenteritis coronavirus genome. J Virol, 2002, 76 11518 11529

26 Kuo L, Masters P S. The small envelope protein E is not essential for murine coronavirus replication. J Virol, 2003, 77: 4597 4608

27 Cyranoskiand D, Abbott A. Virus detectives seek source of SARS in China's wild animals. Nature, 2003, 423: 467

360/csb2003-48-13-1359

(2003-06-03 收稿, 2003-06-26 收修改稿) 